\title{
A blow-up result for the wave equation with localized initial data: the scale-invariant damping and mass term with combined nonlinearities
}

\author{
Makram Hamouda ${ }^{1}$ and M.Ali Hamza ${ }^{1}$ \\ ${ }^{1}$ Imam Abdulrahman Bin Faisal University
}

October 29, 2020

\begin{abstract}
We are interested in this article in studying the damped wave equation with localized initial data, in the $\backslash$ textit $\{$ scale-invariant case $\}$ with mass term and two combined nonlinearities. More precisely, we consider the following equation: \begin \{displaymath } \} $\backslash \mathrm{d}(\mathrm{E}) \backslash \mathrm{hspace}\{1 \mathrm{~cm}\} \mathrm{u}_{-}\{\mathrm{tt}\}-\backslash \operatorname{Delta} \mathrm{u}+\backslash \operatorname{frac}\{\backslash \operatorname{mu}\}\{1+\mathrm{t}\} \mathrm{u}_{-} \mathrm{t}+\backslash \operatorname{frac}\left\{\backslash \mathrm{nu} \mathrm{u}^{\wedge} 2\right\}\left\{(1+\mathrm{t})^{\wedge} 2\right\} \mathrm{u}=\left|\mathrm{u}_{-} \mathrm{t}\right|^{\wedge} \mathrm{p}+|\mathrm{u}|^{\wedge} \mathrm{q}, \backslash$ quad $\backslash \operatorname{mbox}\{\operatorname{in}\} \backslash \backslash \mathrm{R} \wedge \mathrm{N} \backslash \operatorname{times}[0, \backslash \operatorname{infty})$, lend $\{$ displaymath $\}$ with small initial data. Under some assumptions on the mass and damping coefficients, $\$ \backslash \mathrm{nu} \$$ and $\$ \backslash \mathrm{mu}>0 \$$, respectively, we show that blow-up region and the lifespan bound of the solution of $\$(\mathrm{E}) \$$ remain the same as the ones obtained in (missing citation) in the case of a mass-free wave equation, \{ $\backslash$ it i.e. $\} \$(E) \$$ with $\$ \backslash$ nu=0 $\$$. Furthermore, using in part the computations done for $\$(\mathrm{E}) \$$, we enhance the result in (missing citation) on the Glassey conjecture for the solution of $\$(\mathrm{E}) \$$ with omitting the nonlinear term $\$|\mathrm{u}|^{\wedge} \mathrm{q} \$$. Indeed, the blow-up region is extended from $\$ \mathrm{p} \backslash$ in $\left(1, \mathrm{p}_{-} \mathrm{G}(\mathrm{N}+\backslash\right.$ sigma $\left.)\right] \$$, where $\$ \backslash$ sigma $\$$ is given by $\backslash$ eqref $\{$ sigma $\}$ below, to $\$ \mathrm{p} \backslash$ in $\left(1, p_{-} \mathrm{G}(\mathrm{N}+\backslash \mathrm{mu})\right]$ yielding, hence, a better estimate of the lifespan when $\$(\backslash \mathrm{mu}-1)^{\wedge} 2-4 \backslash \mathrm{nu} \wedge 2<1 \$$. Otherwise, the two results coincide. Finally, we may conclude that the mass term $\{\backslash$ it has no influence $\}$ on the dynamics of $\$(\mathrm{E}) \$$ (resp. $\$(\mathrm{E}) \$$ without the nonlinear term $\$|\mathrm{u}|^{\wedge} \mathrm{q} \$$ ), and the conjecture we made in (missing citation) on the threshold between the blow-up and the global existence regions obtained holds true here.
\end{abstract}

\section{Hosted file}

Blow-up-Damped wave-mass-MMAS.pdf available at https://authorea.com/users/44799/articles/ 489611-a-blow-up-result-for-the-wave-equation-with-localized-initial-data-the-scaleinvariant-damping-and-mass-term-with-combined-nonlinearities 

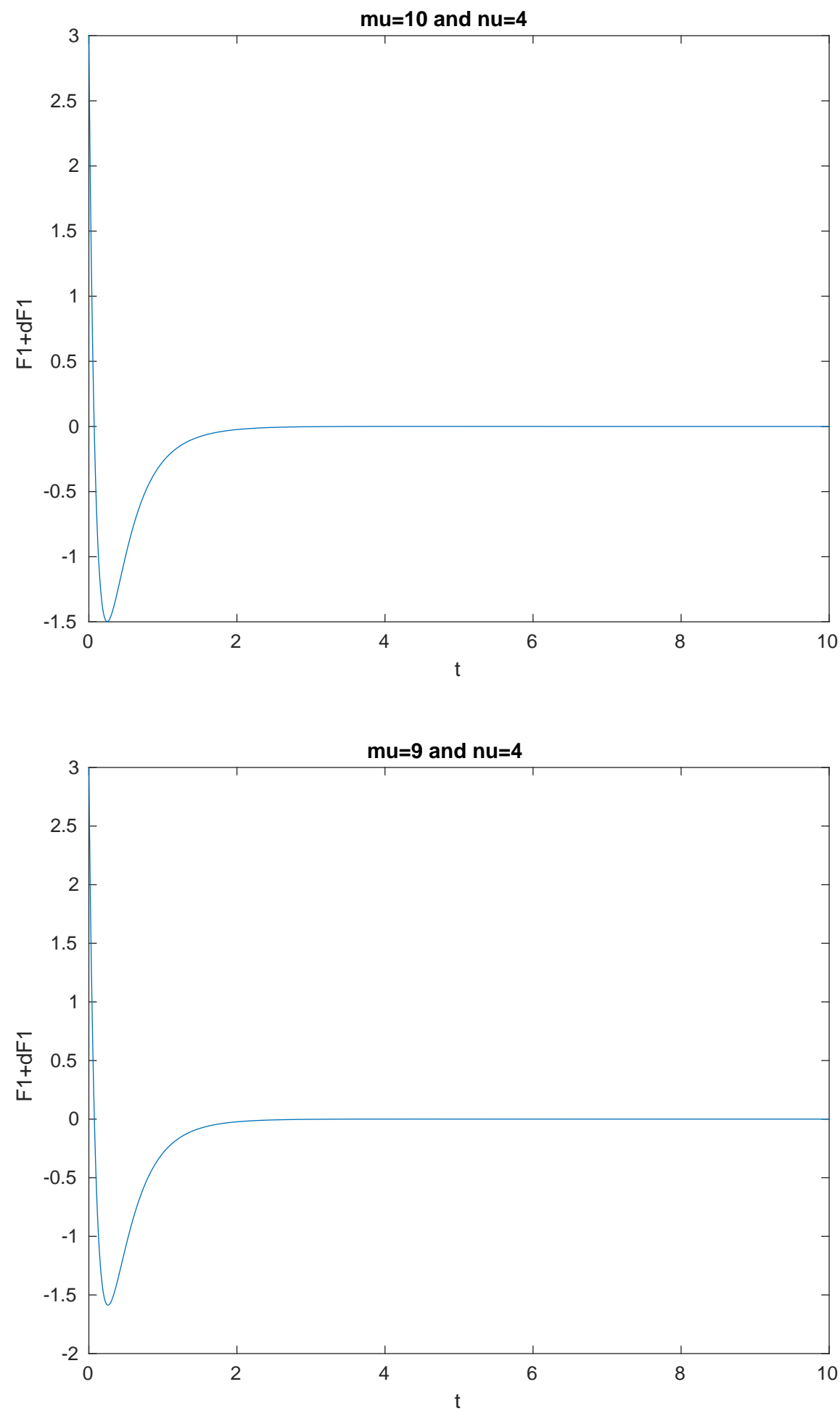

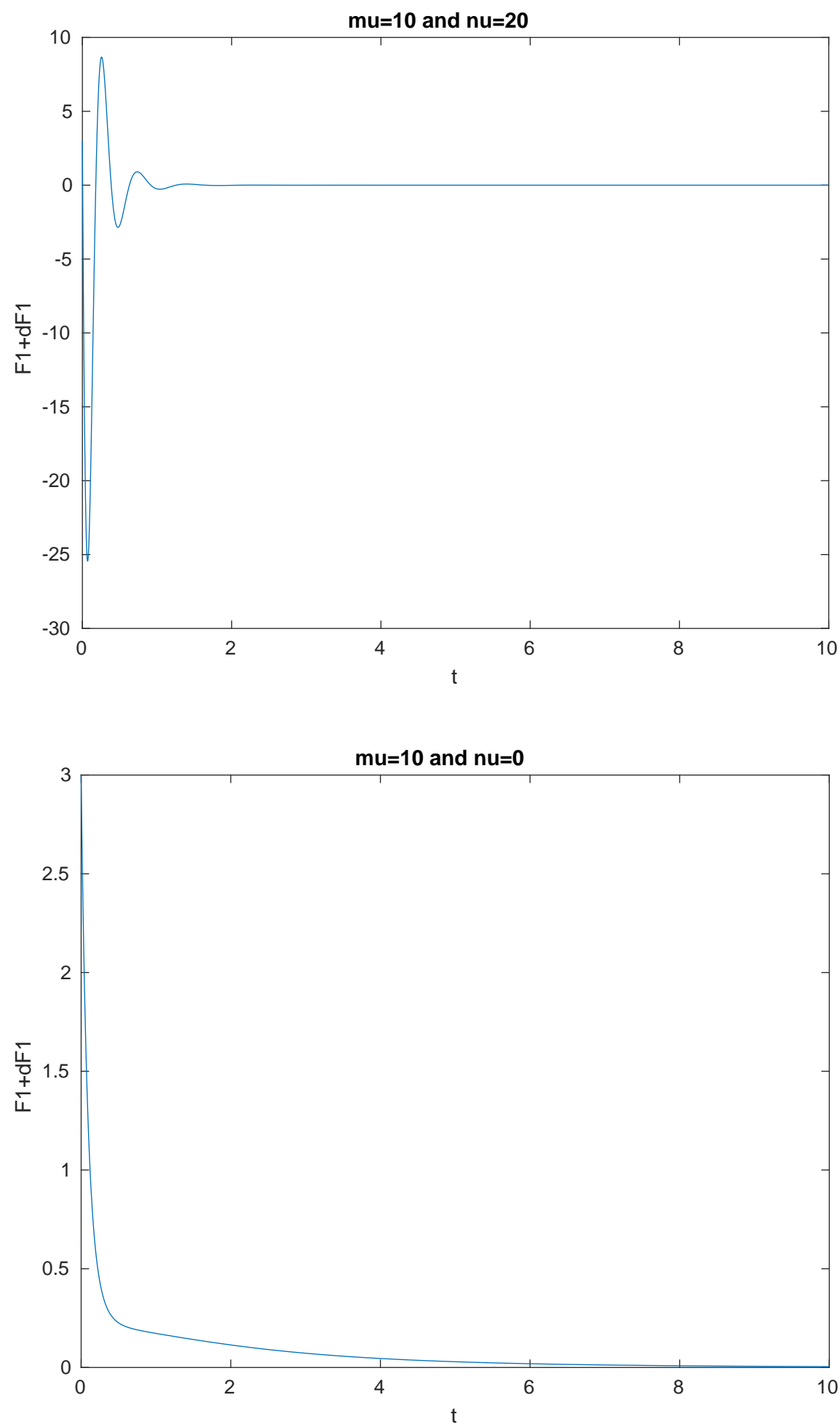

\section{References}

